\title{
Muon detector and muon flux measurement at Yangyang underground laboratory for the COSINE-100 experiment
}

\author{
Hafizh Prihtiadi* \\ on behalf of the COSINE-100 Experiment \\ Department of Physics, Bandung Institute of Technology, Bandung 40132, Indonesia \\ E-mail: hafizh.prihtiadiegmail.com
}

The COSINE-100 experiment is a joint experiment by the KIMS-NaI (Korean Invisible Mass Search) and the DM-Ice with a goal to reproduce the annual modulation signature reported by the DAMA using the same target material. In rare event searches, the cosmic-ray muon can produce the seasonal modulation of events which can mimic weakly interacting massive particles (WIMP)-like signal in nuclear recoils. To tag the muon-events and study the correlations between muon and crystal signals, COSINE-100 has installed muon detectors in the shielding structure at Yangyang Underground Laboratory (Y2L). An array of 3-cm thick plastic scintillator panels surrounds the crystal detector in all sides. This paper describes the assembly of the detector, DAQ, test performance, and the muon flux measured at the experimental site. The detector efficiency is calculated to be $99 \pm 1 \%$ and preliminary result of the muon flux is found to be $344 \pm 1$ (stat.) \pm 34 (syst.) muons $/ \mathrm{m}^{2} /$ day.

35th International Cosmic Ray Conference - ICRC2017

12-20 July, 2017

Bexco, Busan, Korea

${ }^{*}$ Speaker. 


\section{Introduction}

Cosmic-ray muons, created when the primary cosmic rays interact with nuclei in the atmosphere, will produce secondary products decaying into leptons, pions $\left(\pi^{ \pm}\right)$and kaons $\left(K^{ \pm}\right)$. Since muons $\left(\mu^{ \pm}\right)$can easily penetrate to the deep underground laboratory and they can make a background in low-count rate experiment $[1,2]$. Muons can pass through the detector and its surrounding material that produce a secondary backgrounds or spallation neutrons, which can mimic a WIMP-like signal in dark matter experiment [3]. In the past, the study of annual modulation from either muons or muon-induced events have been investigated $[4,5]$. The coincidence of the cosmic-ray modulation might be considered muons as a possible source that can explain the longstand DAMA annual modulation signature $[6,7,8,9]$. The COSINE-100 experiment is the phase-I of the joint effort between KIMS-NaI (Korean Invisible Mass Search) and DM-Ice with a goal to confirm or refute the DAMA-signal [10,11, 12]. COSINE-100 has constructed the muon detector to tag and study the correlations events between crystal and muons data.

\section{Muon Detector Construction and Performance}

The outer detector shielding is consist of 42 PMTs of 2-inch PMT from Hamamatsu Photonics and 37 plastic scintillator with 3-cm thickness of type EJ-200 having maximum light output at wavelength $425 \mathrm{~nm}$. A scintillator is glued to an acrylic light guide and then to a PMT photocathode. Optical cement BC-600 is used for the couplings. To seal the external light and to prevent from physical damage, the scintillator was wrapped by TYVEK reflector, $50 \mu \mathrm{m}$-thick aluminum foil, and black sheet. The general layout of inner and outer detector is shown in Fig. 2. For the detector that is longer than $2 \mathrm{~m}$ with a PMT attached only one side, a Vikuiti reflector is attached to the other edge to have better light collection. On average, the light yield can be improved with the reflector by $10 \%$. These muon counter gains have been adjusted by using the number of photoelectrons in a separate trigger test setup. In order to determine the detector efficiency, four panels have been stacked which top and bottom part have used for a module trigger events. A triggered coincidence event can be used to estimate the muon tagging efficiency of the middle panels that do not account in the trigger. Events with amplitude more than 20,000 ADC count are selected as a muon-like events. This restriction is useful for rejecting background events which are not counted as a muon. The efficiency is defined as the number of pulses detected by the PMT compared to the number of muons that actually passed through the module trigger. On average, the muon tagging efficiency is estimated to be at least $99.5 \%$ with systematic error of $0.5 \%$. The error can be explained as the edge effect and misalignment, when the muon particle with large zenith angle would pass the edge of the top or bottom panels but penetrate the whole thickness of middle panel. This edge effect is considered as a systematic error of detection efficiency correction. We estimated the muon-flux at ground level to be $136 \pm 7$ muons $/ \mathrm{m}^{2} / \mathrm{s}$.

For selecting muons, we require the coincidence events between two panels. From the charge spectrum with the selected muon events, the number of photoelectrons is estimated after requiring a threshold and fitting by Landau function. 


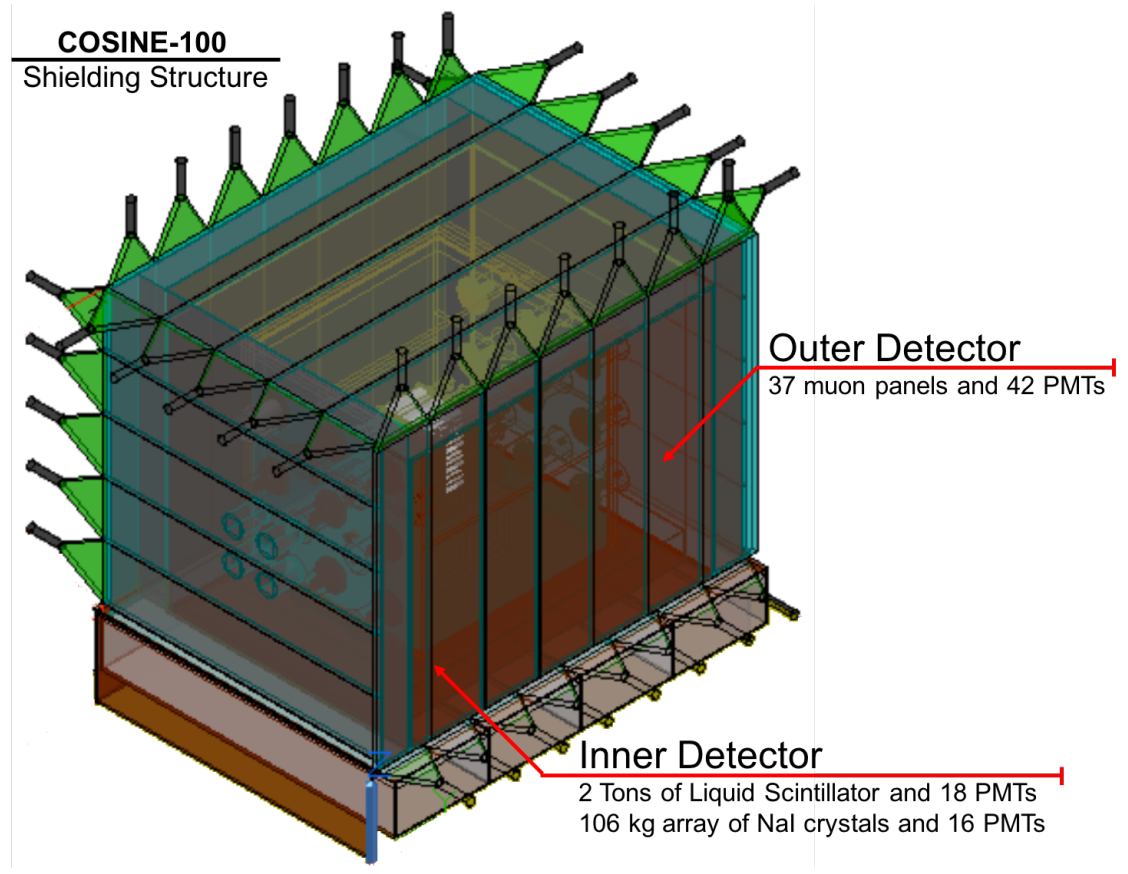

Figure 1: A sketch of the COSINE-100 shielding structure

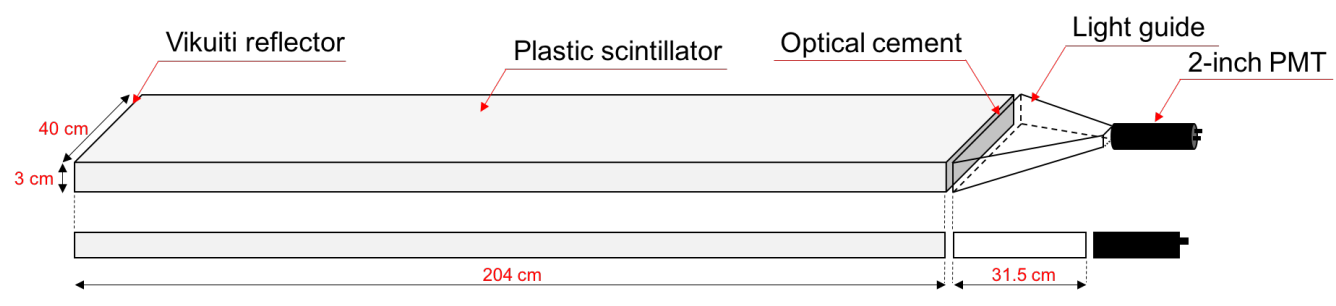

Figure 2: Schematic view of muon detector materials and assembly part

\section{Muon Detector at $\mathrm{Y} 2 \mathrm{~L}$}

Y2L is located in the northeast of Seoul Korea, $200 \mathrm{~km}$ east of Seoul. The Y2L facility has two experimental areas, A5 and A6 tunnel, which are accessed by a car through a $2 \mathrm{~km}$ driveway. The COSINE experiment is located in one of the recently established labs in the A5 tunnel, while the KIMS experiment is located in the A6 tunnel.

\subsection{DAQ and Data Structure}

The total of 60 signal channels (18 channels from the liquid scintillator and 42 channels from the muon detector) is digitized at $64 \mathrm{MS} / \mathrm{s}$ Flash-Analog to Digital Converter (FADC). We developed two parameters to determine the muon signal. Firstly, we set the integrated charge threshold more than 4,000 ADC count (equivalent to approximately $763 \mathrm{pC}$ ). Second, a coincidence window with a $400 \mathrm{~ns}$ of the coincidence hits from at least two PMTs (either two PMTs at muon detector or at least one PMT at LS Veto and one PMT at muon). If the signal is satisfied with two conditions, further the M64ADCs send a trigger signal to the Trigger Control Box (TCB) and collect the data at 
$4 \mu$ s gate window. Within the gate window, M64ADC integrates charge and saves time information when the PMT has fired.

\subsection{Muon Event Selection}

Muons can deposit large amounts of energy when they passes the detectors. This high energy cosmic-ray muon can be selected by applying selection criteria. By applying these requirements most of the non-muon events or backgrounds, are rejected while the loss of efficiency is almost negligible. Fig. 3 (a) is shown the integrated charged of top panel detector after digitized from the DAQ. We require the coincidence events between any combination of two panels. This technique can determine the region for respective part of the spectrum, which is either region for low energy background or high energy for muons. A charge threshold cut at 14,000 ADC count is applied after the coincidence condition is required.

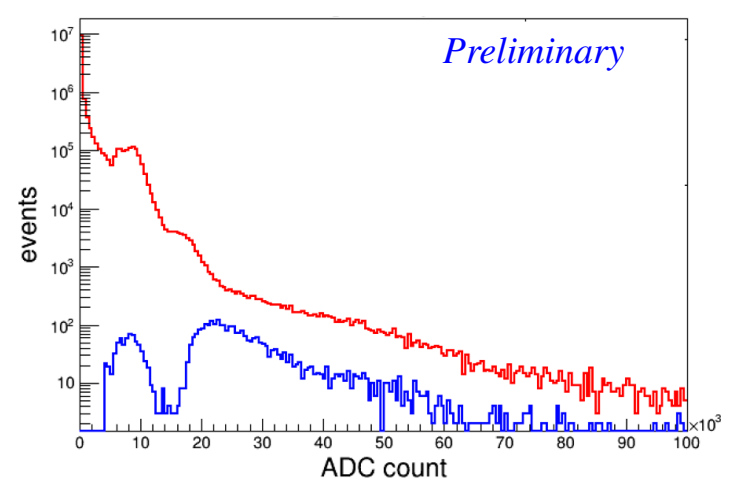

(a)

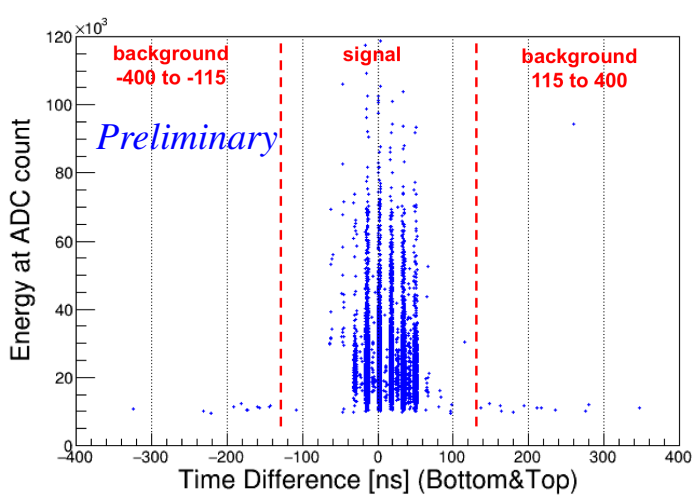

(b)

Figure 3: (a) Distribution of energy depositions in a top panel. The solid red line shows total integrated charge of the top panel. Blue line data is selected by requiring two panels in coincidence, (b) Time difference of PMT signals at Top panel module in range from -400 ns to $400 \mathrm{~ns}$, selected with a two module coincidence cut.

The muon coincident signal is shown in Fig. 3 (b), a $\Delta$ t distribution of coincidence events of Top and Bottom panel. In contrast, muon-like events show a much more confined in distribution of time differences within the gap of signal is $16 \mathrm{~ns}$ time resolution in M64ADC DAQ. The signal distribution is well performed by Gaussian fitting and selecting signal region by statistical significance of $5 \sigma$. Here, we defined to signal region in range from -60 ns to $80 \mathrm{~ns}$ region for muon-like events. Considering different cable lengths, we apply the time difference cut in slightly wider range from - 115 ns to $115 \mathrm{~ns}$. In this distribution, an accidental background coincidence are occurred in low energy region. We counted the total random-events in outside of signal region and estimated them in the signal region. On average, the fake-event rate is found to be $0.1 \%$.

We derived the detection efficiency of the muon detector by using Top module and LS Veto as a trigger detector. A triggered coincidence event is used to estimate the muon in the Bottom module. Fig. 4 is shown the spectrum of muon-like events. Here, a small fraction of external background is shown contribute at high energy region which can be estimated to be $1 \%$. Overall, the detector efficiency is found to be $99 \pm 1 \%$. About $1 \%$ of muon entering the detector volume 
is undetected and this is due to a gap in between the detector about $2 \mathrm{~mm}$ and made some muons missed counting which pass through a panel.

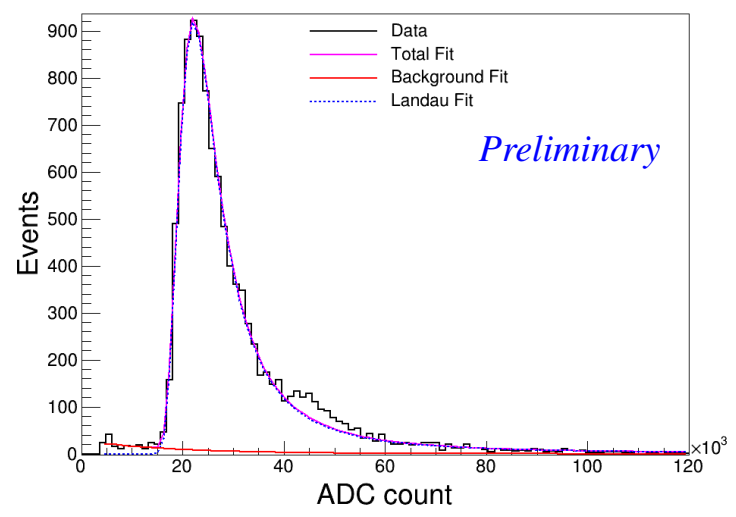

Figure 4: Spectrum of muon-like events with a Landau fit. Below the 14,000 ADC counts, accidental coincidence due to external gamma events happens more frequently. the blue dotted line is from muon-like events after fitting with a Landau function excluding backgrounds at low energy.

\subsection{Muon Flux at Y2L}

Since Y2L has 2 experiment areas with different depth, which are A6 for KIMS Experiment and A5 for the AMoRE (Advanced Mo-based Rare process Experiment) and the COSINE Experiment [13]. The laboratory areas have a minimum granite overburden of $700 \mathrm{~m}$ where cosmic-ray muon flux are measured to be $2.7 \times 10^{-7}$ muons $/ \mathrm{cm}^{2} / \mathrm{s}$ or $233 \mathrm{muons} / \mathrm{m}^{2} / \mathrm{s}$ in A6 [14]. The muon flux is calculated as the ratio between the selected muon, the detector effective surface area, and total live time of analyzed data (more than $6 \times 10^{5}$ selected muons after cut).

$$
\phi\left(E_{\mu}\right)=\frac{N_{\mu}}{t_{d} \cdot A_{d} \cdot \varepsilon_{\mu}}
$$

Where $N_{\mu}, 394,121$ muon-like events with energy deposition greater than 14,000 ADC count, is the number of muons after selection criteria and across the entire top-area, $t_{d}=218.6$ days, is the live time of the detector, $A_{d}=5.24 \mathrm{~m}^{2}$, represents the detector effective area ( $2.62 \mathrm{~m}$ in length and 2 $\mathrm{m}$ in width), and $\varepsilon_{\mu}=99.9 \%$, stands for the detection efficiency of muons after taking into account the saturation of detector and the muon energy loss through radiative process. The water equivalent depth of Y2L is estimated to be $1,850 \mathrm{~m}$ as the product of the measured average rock density of $2.7 \mathrm{~g} / \mathrm{cm}^{3}$. This result of Y2L is consistent with slant-depth muon flux. The muon flux was obtained by dividing the measured counts by the measurement time and the detector area of 5.24 $\mathrm{m}^{2}$. The geometrical uncertainty, the inefficient area of top panel detector was estimated as $10 \%$. Requiring a module being hit in at least two sides, we accumulated 18,503 muon candidates events after applying selection cut which corresponds to a measured rate to be $344 \pm 1$ (stat.) \pm 34 (syst.) muons $/ \mathrm{m}^{2} /$ day. Fig. 5 shows our result comparing with the slant-depth model which describes the cosmic ray muon flux of others underground laboratories at different depth. 


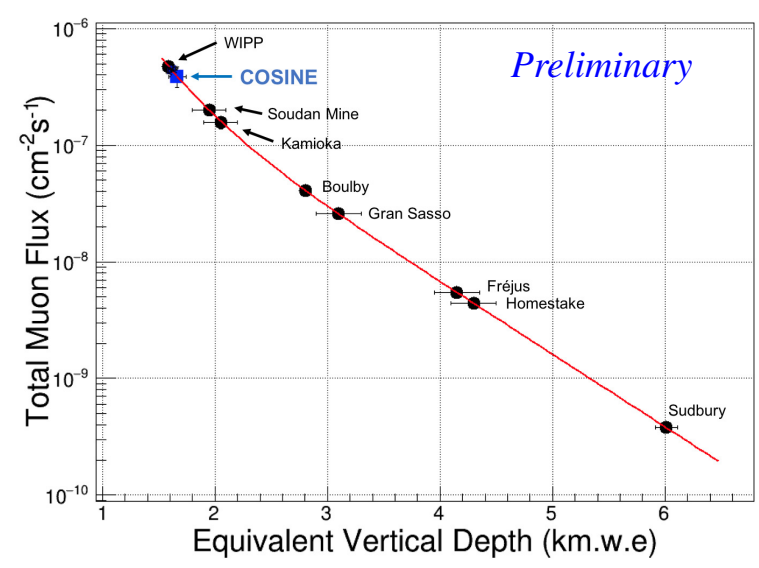

Figure 5: The Y2L measured and compared with the fluxes measured at other underground laboratories.

\section{Summary}

The COSINE-100 Experiment has been installed an array of muon detector on the outside of the detector shielding. The purpose is dedicated to tag and study the correlations of the stream data between crystal and muon events which can mimic the WIMP-like signal. Several test have been done to validate performance of the detector. The detector efficiency is measured to be $99 \pm 1 \%$ and the muon flux to be $344 \pm 1$ (stat.) \pm 34 (syst.) muons $/ \mathrm{m}^{2} /$ day. The flux at the $\mathrm{Y} 2 \mathrm{~L}$ is measured and consistent with the results obtained in other underground experiments sites. Currently, the data taking is still continuing to get sufficiently large statistics.

\section{Acknowledgments}

We thank the Korea Hydro and Nuclear Power (KHNP) Company for providing the underground laboratory space at Yangyang. We also acknowlege the following supports : the Institute for Basic Science (IBS) under project code IBS-R016-A1, Republic of Korea; the Alfred P. Sloan Foundation Fellowship, NSF Grants No. PHY-1151795 and PHY-1457995, WIPAC, the Wisconsin Alumni Research Foundation, and Yale University, United States; STFC grant ST/N000277/1, United Kingdom; CNPq, Brazil.

\section{References}

[1] T. K. Gaisser, Cosmic Rays and Particle Physics, Cambridge University Press (1990).

[2] K. Olive et al., Chin. Phys. C38 (2014) 09001.

[3] D.-M. Mei et al., Phys. Rev. D 73 (2006) 053004.

[4] J. H. Davis, Phys. Rev. Lett. 113 (2014) 081302.

[5] A. S. Malgin, J. Exp. Theor. Phys. 121 (2015) 212.

[6] D. Nygren, ArXiv:1102.0815 (2012).

[7] R. Bernabei et al., Eur. Phys. J. C 67 (2010) 39. 
[8] R. Bernabei et al., Eur. Phys. J. C 73 (2013) 2648.

[9] R. Bernabei et al., EPJ Web Conf. 70 (2014) 00043.

[10] J. Cherwinka et al., Phys. Rev. D 90 (2014) 092005.

[11] P. Adhikari et al., Eur. Phys. J. C 76 (2016) 185.

[12] K. W. Kim et al., Astropart. Phys. 62 (2015) 249.

[13] H. S. Lee et al., Phys. Lett. B 663 (2006) 201.

[14] J. J. Zhi, High Energ. Phys. Nucl. 29 (2005) 8. 